\section{Military Technical College Kobry El-Kobbah, Cairo, Egypt}

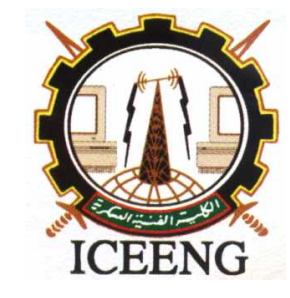

\title{
Motor drive design in database environment
}

\author{
By \\ Juhan Laugis* \\ Valery Vodovozov**
}

\section{Abstract:}

The problem of electric drive design concerns the multi-criteria non-linear problems having a number of solutions. In daily practice the search of optimum solution bases a great deal on the designer intuition and experience. Therefore, multiple obtained results have a low degree of equipment use, unwarranted complexity, or excessive cost. Proposed approach is devoted to implementation of the novel expert system into the design stages concerned the work with databases of the drive components. Examples of effective algorithms are given to choose an optimum ratio of electrical and mechanical components. The soft tool is developed to generate and edit structured query language sentences, to extract information from databases, as well as to suit multitude sets for particular applications.

\section{Keywords:}

Design, electric drive, expert system, modelling, simulation, software

\footnotetext{
* Tallinn University of Technology, Estonia

** St. Petersburg Electrotechnical University, Russia
} 


\section{Introduction}

An electric drive belongs to inhomogeneous, nonlinear, and non-stationary class of systems [1]. Object heterogeneity reflects in various natures of structural components and assemble of informational and energetic processes. Heterogeneity deals with analog and discrete nature of different inputs and variables. Power pulse generation with continuous electromechanical transformation and discontinuous informational conversion is referred to the same processes as well. When the reaction on different inputs is discussed, an electromechanical system is devoted to non-linear group. Nonlinearity is produced by the limited controllers and sensors code lengths, as well as by technical and program non-linear chains and blocks. They course amplitude modularity of digit-to-analog transformation, non-regular signal shapes, and semi-controllability of power converters, saturation in magnetic chains of electric machines, plags, gaps, backslashes, Coulomb and fluid friction of mechanical gears and actuators. A nonstationary property of electric drive is a result of changing parameters of the system and varying load torques and moments of inertia of mechanical system. This feature is taking into account in description of objects behavior as time-dependent statements.

Therefore, an electric drive design procedure involves a number of complex problems [2]. Some of them are:

- timing calculation and the mechanism travel diagram construction;

- computation of mechanism forces and torque/power patterns synthesis;

- gear dimensioning and selection;

- motor dimensioning and selection;

- optimum motor-gear composition and checking;

- power electronic converter dimensioning and selection or design a novel one;

- design of controllers for multi-loop control and adjustment;

- process simulation with building of steady-state diagrams and transients;

- development of the drive documentation;

- economical basing and efficiency evaluation.

Effective assembling of the drive systems meets with the problems of such kind owing to rather complicated algorithms [3]. Today, the three ways of the drive design are used in the designers' community.

The first of them starts from the selection of a manufacturer; further the manufacturer design technology and recommendations is followed. To compute and select equipment, the leading companies have developed their specific technologies. Examples are the 
guides and software of "Siemens" [4], "Omron", "Sew Eurodrive" [5], "Maxon Motors", "Mitsubishi Electric", etc. Such approach is usual for the majority of firms that carry out project designs and have rich experience in acceptance of the decisions on the basis of extensive computer databases, coming up to numerous catalogue archives and "absorbing" their contents and structures. The main its drawback is the technological restriction and data limiting that deprive a designer of optimum way in the project. It is especially important in the first stage, when the most responsible decisions are decided.

The novel way has been developed and described in [6], [7]; it starts from the traditional load computation using the designers' own experience and methods. Further a designer selects a group of gear families and types of different companies using the load calculation results. Through these gears, the forces and mechanism speed found before will be converted to the equivalent values on motor shaft. Then, for each gear type the own motor type is to be picked out, counted on the converted forces and speed. Thus, hundreds and thousands sets of possible drive variants are generated. Once the equipment framework will be found, the new problem appears: which of the suitable motor-gear combinations are optimum? To find a solution, a designer can form appropriate criteria and sort them. It may be a criterion of maximum accuracy or speed, minimum weight, power, or inertia, highest rigidity, etc. This way, the full scale of the electromechanical and electronic properties is gathered, from which the choice is done on the basis of judgments about preferences of that or other criterion.

An approach proposed in this paper bases on the automatic multitude simultaneous gear-motor-converter assembles calculation and selection with analyzing and sorting them on the judgments about the criterion preferences.

\section{Query language to process databases of the drive components}

Different manufacturers worked out hundreds and thousands of databases that help in providing most of stages described above, short of first and last one. A specialist in electric drive design receives next input specifications from these data sheets:

- Parameters of motors with various action and construction principles, powers and speeds. Each motor is specified by its rated torque, moment of inertia, mass, efficiency and a list of electrical values.

- Specifications of transmissions distinguished by design and construction, power and ratio. Each gear-box has its rated and maximum torque, primary and secondary speeds, moment of inertia, mass and efficiency.

- The nameplate data of industrial electrical power converters with ac and dc principles and different powers. Each record includes rated and maximum voltages, currents, frequencies, and efficiency. 
All these data sheets are prepared by various manufactures using different languages and operation systems. They have multiple structures, data types, data fields, keys, names, and inter-sheet links. Not all required data are published into the sheets; some of them present the know-how of manufacturers hided from users. Often, information carriers advertising nature, which is not needed to derive a design. Some data are published in Internet; other is obtainable through printed materials and journals.

To overcome these problems and obtain an access to the required information, a novel expert system based on the universal database has been proposed and described data sources were connected to this joined database. Unlike the known simulation tools, for example [8], the proposed package is deeply integrated with database equipment. For the selected connection, specific software has been developed [9]. The uniform structure, data types, keys, and tables were prepared also. Then using structured query language (SQL), a number of templates were developed, in which calculated data should be substituted to find the sets of the drive components.

A query language gives a set of rules and tools that helps user to search required information into the databases. As a query answer, a database management system replies a result in the form of screen (virtual) table displayed a recordset of a certain structure, which may be used to build a real summary table, report, or a new query. Today, SQL is an international standard of query languages used in all databases used all-round the world. The data search is not the only operation of SQL. The language provides tables' creation, control, connection, and edition as well. SQL grammar bases on SQL statements, each included variables, which are the names and values of particular tables, fields, and keys. For example:

\section{SELECT * FROM csCONTROLTECHNIQUES}

WHERE Pc_W $>900$

AND Ic_A $>21$

ORDER BY Pc_W

This statement searches all records in the table csCONTROLTECHNIQUES, which satisfy the requirement that the candidate converter power must exceed 900 and current must exceed 21 whilst the obtained records have to be ordered alphabetically on the screen.

The next statement searches information in three tables simultaneously with online calculation of dependant parameters. Here, gears, motors, and converters are selected together matching powers $\left(\mathrm{P}_{-} \mathrm{W}\right)$, torques $\left(\mathrm{M} \_\mathrm{Nm}\right)$, rotational and linear speeds $\left(\mathrm{n} \_\mathrm{rpn}\right.$, vg_cms), moments of inertia (J_kgcm2), and currents (Ic_A) together: 
SELECT $*$ FROM gs, msSEW, csCONTROLTECHNIQUES

WHERE vg_cms $/ 100>=0.1$

AND Pg_W $* 100 /$ vg_cms $>=671 / 0.1$

AND M_Nm $>671 /$ ng_rpm $* 10 /[\%$ g] $* 100$

AND J_kgcm2 / $10000>0.004 / 10$

AND n_rpm $/ 10>59.5$

AND Pc_W $>15 * 60$

AND Ic_A > $14.9 *$ I_A / M_Nm

ORDER BY Pg_W

\section{Database management system}

Further, the soft tool has been developed to build the necessary SQL statement templates and to insert the arguments into this templates. The proposed software includes the comfortable Windows interface with dialog boxes and SQL edition environment. The required databases link to the package automatically or manually. The first tab of the database window involves the list of tables used to find data as well as the detailed data sheets with the drive components' records. The developed SQL editor has all the needed instruments to write, copy, cut, paste, delete, insert, and check the language components and SQL statements. Further to the query running, the combined result is obtained, where the matched sets of motors, gears, and converters are ranged on the preferred order.

All information selected using SQL may be inserted into the drive model to suit their correctness and efficiency in the common gear-motor-converter dataset. To execute simulation, the three tabs display simulation result: Dynamics, Statics, and Analysis. Any change of model provides the result updating when it activates. Here, the tab Dynamics represents the speed transients of the mechanism and the torque or the current transients of the motor. The values of variables one may measure in the areas where the curves are crossed by the vertical line of cursor. The program indicates them in the status bar. The tab Statics displays the idealized speed-current or speed-torque relations in the open-loop system. Simulation analyses screen includes:

- table of torque, current, and speed instantaneous values per time;

- summary of maximum, minimum, and steady-state values of variables;

- the data, calculated in the process of simulation;

- summary of service factors of the drive equipment that is calculated as the steady-to-rated (in the case of the step signal) or rms-to-rated (in other cases) values ratio: 
- if the motor rated power has been specified in the database, the motor power service factor is obtained as the product of motor torque and motor speed divided by motor power, and the motor torque service factor is obtained as the ratio of steady (rms) torque value and the quotient obtained when rated power is divided by motor speed;

- if the motor rated torque has been specified in the database, the motor torque service factor is obtained as the ratio of the steady (rms) torque and the rated torque values;

- if the motor rated current has been specified in the database, the motor current service factor is obtained as the ratio of the steady (rms) current and the rated current values;

- if the converter rated power has been specified in the database, the converter power service factor is obtained the ratio of the motor torque-speed product and the rated converter power;

- if the gear rated power has been specified in the database, the gear power service factor is obtained as the ratio of the load torque-speed product and the gear rated power;

- if the gear transmission ratio and rated torque have been specified in the database, the gear torque service factor is obtained as the product of the steady (rms) torque and transmission ratio divided by the gear rated torque.

The selected components may be used in the project since the simulation results satisfied the designed requirements. If the results are unsatisfactory, the new SQL queries have to be created to continue the search of optimum drive components.

\section{Conclusion}

A novel expert system to search the optimum components of the designed motor drive has been proposed. This approach is based on the implementation of the SQL into the design stages concerned the work with databases. Examples of effective algorithms are given to choose an optimum ratio of electrical and mechanical components. The soft tool is described to generate and edit SQL sentences, to extract information from databases, as well as to test different component sets for particular applications. Obtained results help to increase the equipment service factors and to avoid unwanted complexity and excessive motor drive cost. 


\section{References:}

[1] V. Vodovozov, Theory and Systems of Electric Drives. ETU Publishing, St. Petersburg, 2004. ISBN: 5-7629-0567-5

[2] S. Cetinkunt, Optimal Design Issues in High-Speed High-Precision Motion Servo Systems. Mechatronics, 1991, Vol. 1(2), P. 187-201.

[3] F. Roos, H. Johansson, and I. Wikander, Optimum Selection of Motor and Gearhead in Mechatronic Applications. Mechatronics, 2006, Vol. 16(1), P. 63-72.

[4] Siemens Standard Drives. Application Handbook, Congleton, 1997.

[5] Drive Engineering - Practical Implementation. Volume 1. Drive Arrangements with SEW Geared Motors. Calculation Methods and Examples, SEW Eurodrive, 1998.

[6] V. Vodovozov and A. Loparev, Simulation Tools for Design and Testing of Electric Drives, 10th International Power Electronics and Motion Control Conference EPE-PEMC-2004, Riga, Latvia, 2004, Paper DS 7.16.

[7] V. Vodovozov and J. Laugis, Object-Oriented Electric Drive Development Technology, IEEE International Electric Machines and Drives Conference IEMDC'07, Antalya, Turkey, 2007, Paper AF000434.

[8] N. Ergodian, H. Hemao, and R. Grisel, A Proposed Technique for Simulating the Complete Electric Drive Systems with a Complex Kinematics Chain. IEEE International Electric Machines and Drives Conference IEMDC'07, Antalya, Turkey, 2007, Paper AF014095.

[9] V. Vodovozov, E. Vodovozova, and E. Tsvetikov, Object-Oriented Models of Electromechanical Systems. 8th European Simulation Symposium "Simulation in Industry", Italy, 1996. 